\title{
Isolation and Identification of Dermatophytesis and Fungal Factors Samples from Infected Patients in Basra - Iraq
}

\author{
Ahmed Hamid Dawood Alatbee ${ }^{1}$, Entiha abdulzahra abdulkareem², Shafeeqa \\ Abdusayed Obaid ${ }^{3}$ \\ \{ahmed.dawood@stu.edu.iq ${ }^{1}$, intihaa.abdulkareem@stu.edu.iq ${ }^{2}$, Shafeeqa \\ .abdulsayed@stu.edu.iq $\left.{ }^{3}\right\}$
}

Southern Technical University, Technical Institute of Basra Department of Anesthesia and Medical Laboratories Techniques, - Iraq ${ }^{1,2,3}$

\begin{abstract}
Fifty samples were collected from patients who showed symptoms of Fungal infection in private clinics and government hospitals. After examining, samples found 20 patients with fungal skin. The study showed an increase in dermatophytosis at an earlier age, as well as increased rates of infection in more rural areas compared to downtown. Where the study confirmed a higher rate of Tinea infections was Tenia corperis $50 \%$ followed by after that Tenia pedis $25 \%$ while the lowest rate of infection tinea capitis $15 \%$. While two infections appeared by pityrs Versicolor is.
\end{abstract}

Keywords. Microsporum, Dermatophytes, candidia spp ,Tenia infection, Trichophyton spp.

\section{Introduction}

The recent incidence of tinea pedis and the spread of one major causative agent, T. rubrum, increased. It is usually not taken care of despite its great impact on the environment and human health.[1] Dermatophytes are a group that can invade the keratinized tissues (hair, skin, and nails) of humans and other animals to produce an infection known as dermatophytes, commonly referred to as a ringworm. [2] Fungal skin infection in the general population of developing countries The World Health Organization has reported a prevalence of up to $19.7 \%$ [3] Some species (e.g., Epidermal- phyton floccosum, Trichophyton rubrum, Trichophyton mentagrophytes, ) are distributed worldwide, whereas others have a partial geographic restriction (e.g., Trichophyton Sudanese, violaceum, Trichophyton schoenleini, Trichophyton Trichophyton concentric,) [4] Some fungal pathogenic fungi are free to live and cause disease through the entry of their spores through wounds or inhalation and others are part of the normal flora of the human body such as, yeast, Candida. [5] Droplet's cause damage due to toxins and the enzymes they produce because they provoke an allergic reaction and penetrate directly into the tissues [6] The epidemiology of tinea capitis and tinea pedis (with onychomycosis) experienced the most dramatic changes. Changes in the prevalence of tinea corporis, tinea corporis, tinea corporis, and tinea cruris have been less extensively studied. Reflects the spread of dermatophytes responsible for causing local 
tendencies in the head and ringworm, which is believed to be the source of infection. [7] . Sometimes the infection spreads to the skin in other parts of the body (or may have started for the first time in another area, such as sports foot (foot feed.) Usually deepens fungal infection in the body deep in body and is not serious [8].

\section{Materials and Method}

Samples were collected from skin clinics and government hospitals

\subsection{Materials}

1. Wire Loop

2. Potassium Hydroxide Has A Concentration Of $10 \%$

3. Slides

4. Baker

5. Benzene Burner

6. Small Size Shower

7. Stain

8. Stick

9. Swap

10. Cover Slid

\subsection{Method}

\subsubsection{Preparation of Sabouraud Dextrose Agar (SDA)}

1. Suspend $65 \mathrm{~g}$ of the medium in one litter of distilled water

2. Heat with frequent agitation and boil for one minute to completely dissolve the medium.

3. Autoclave at $121^{\circ} \mathrm{C}$ for 15 minutes.

4. Cool to 45 to $50^{\circ} \mathrm{C}$ and pour into petri dishes or tubes for slants.

Hair or skin scrapings were inoculated on the sterilization (SDA '(SDA+Cycloheximide +chloramphenicol then incubate at $30 \pm 1$ o $\mathrm{C}$ and examined every 2-3 days.

\section{Result and Discussion}

Fifty samples were collected from patients who showed symptoms of Fungal infection in private clinics and government hospitals. Fig 1 it found Positive cultural examination 40\%people infected with various ages fungal skin infection while the negative cultural examination was $60 \%$. Fig 2 Dermatomycosis infections in the age group (1-10) highest $40 \%$ followed by age group (11-20) years which was (30\%) than age group (21-30) years then age group(31-40) and age group (41-50) each was (10\%). The study also showed that women are more affected than men. Fig 3 show that the higher rate of tinea infections was tenia corperis $50 \%$ followed by tenia pedis $25 \%$ while the lowest rate of infection tinea tenia capitis $15 \%$. While two infections appeared by pityrs Versicolor. Fig 4 shows that the rates of infection with dermatophytes in males 30\% Were less than females 70\% Which matches the results that have been reached by Kaddouri (2008) [9]This result was not near that found by Tan (2005) [10], which found that the incidence rate for females was higher males in Tikrit[9]. The study also found an increase in the rate of fungal infection in rural areas compared to the city center, where $75 \%$ of the total infections were found in rural areas where culture and health care were low, while $25 \%$ of the total infections were found. In the city, this indicates a very important difference between the level of infections between the countryside and the city and the need to focus on raising the level of health and medical culture in rural areas to avoid fungal infections Fig 5. 


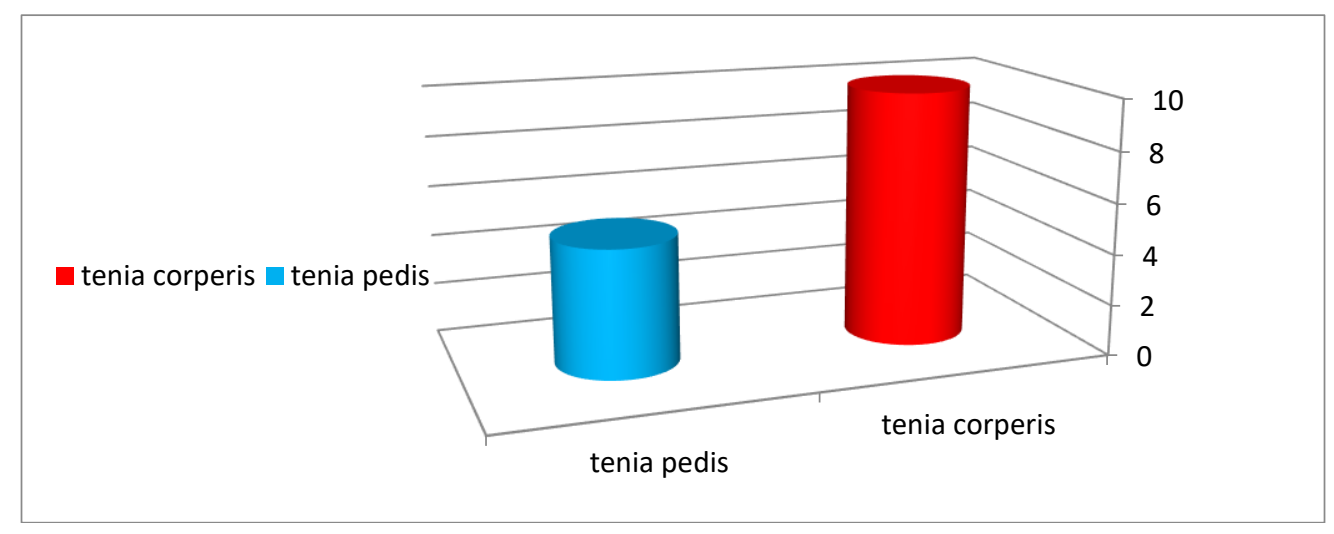

Fig 1. Show the percentage of positive and negative infections.

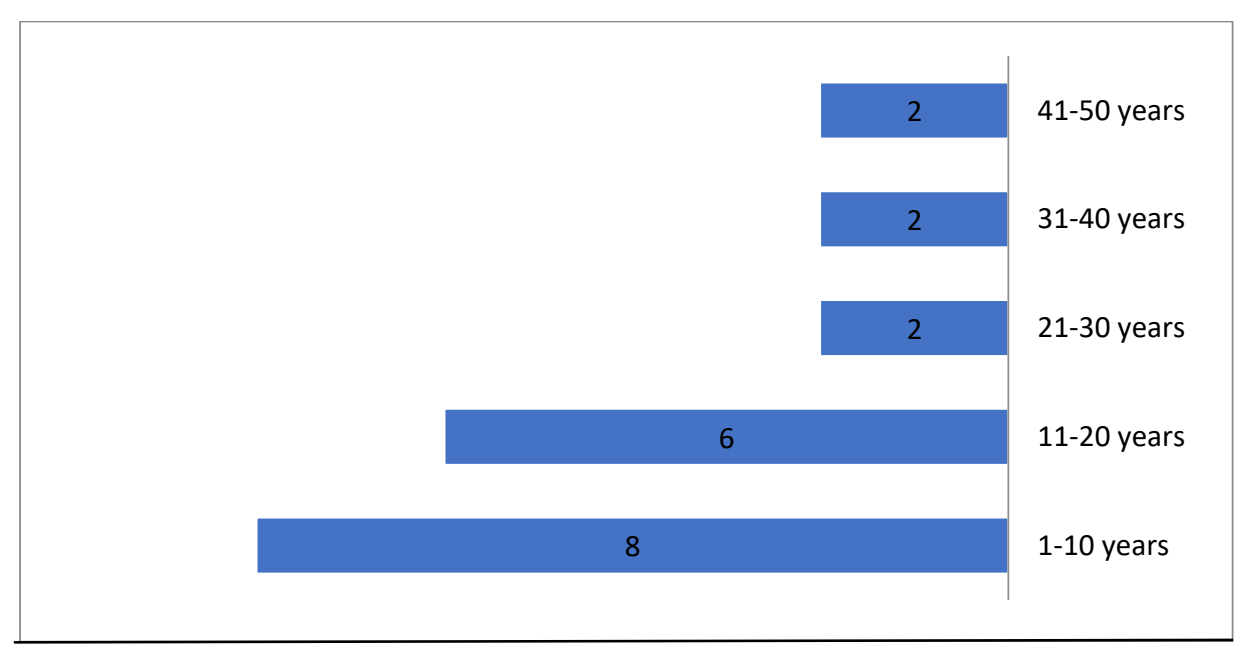

Fig 2. Show the percentage of dermatophytes Infections at different age groups. 


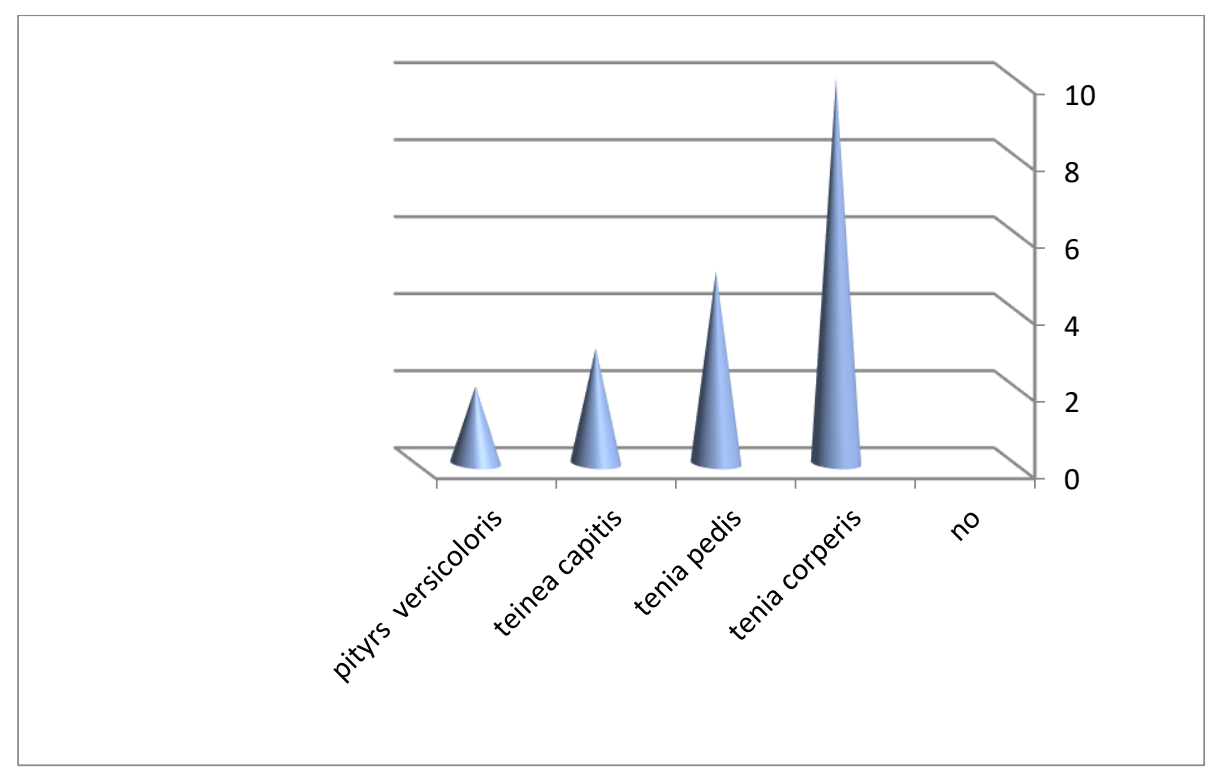

Fig 3. Show The percentages of dermatophytes (tinea) Infections.

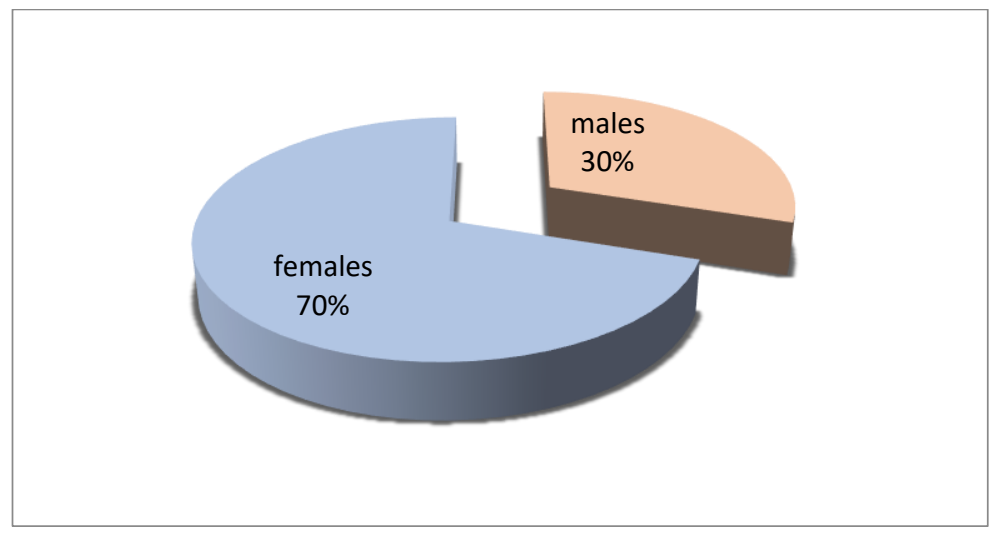

Fig 4. Show that the infection rates with dermatophytes in males and females 


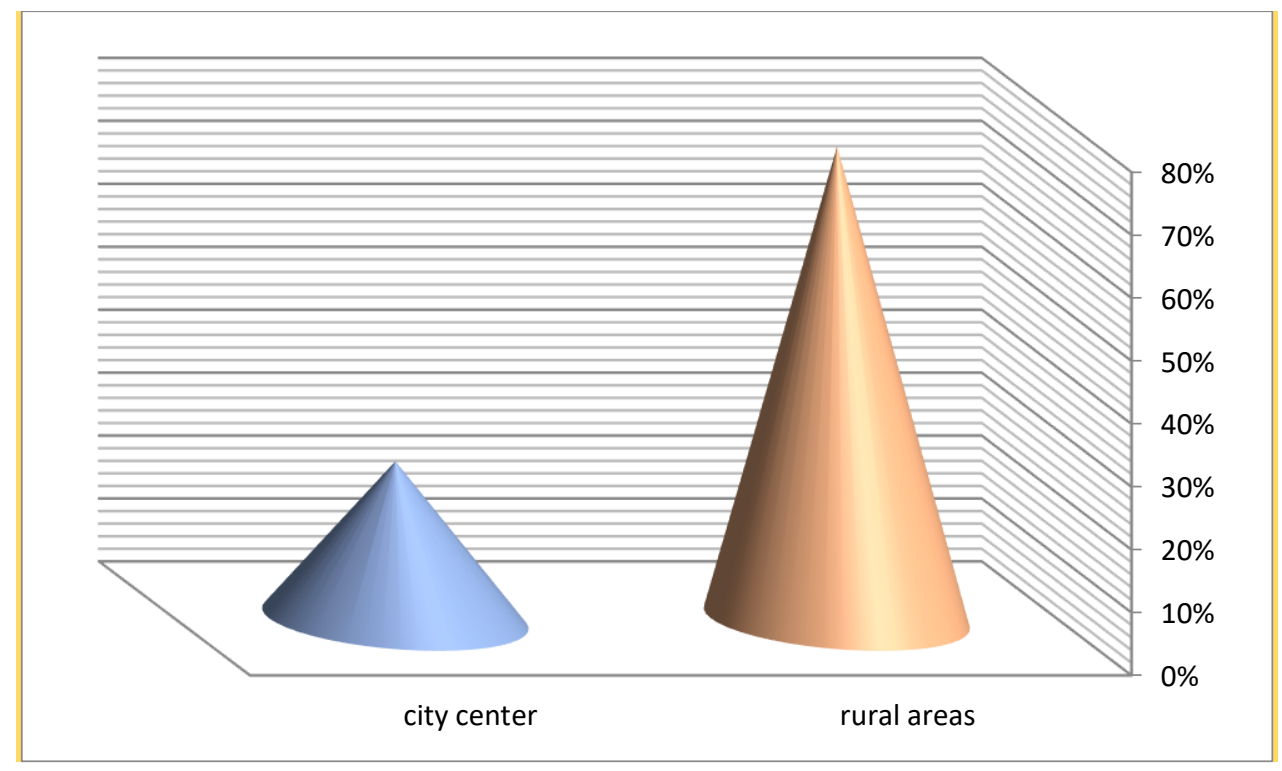

Fig 5. It explains the difference between rural areas and the city centre

\section{Conclusion and Recommendation}

The most important conclusions of the research: Determine the rate of infection with skin fungi and the most types of fungi that affect the skin in Basra Governorate, in addition to determining the most affected age groups, which has a very important role in reducing infection rates. One of the most important recommendations that we recommend in the future is to expand the scope of the study to include all districts and sub-districts in Basra, due to the increased infection of skin fungi, which may be transmitted from one person to another in Basra.

\section{References}

1. Jonathan B. Losos, Kenneth A. Mason, Susan R. Singer, Peter H. Raven, George B. Johnson, McGraw-Hill : biology book : Publisher Obeikan Publishing, 2014 : Number of pages 1378 pages

2. Dr. Harish C. Gugnani : Department of Medical Mycology : Vallabhbhai Patel Chest Institute,University of Delhi, Delhi-110007, India : (2000)

3. Kieran Sweeney and Iona Heath; address : British joumal of General practice .

4. Affiliations : Fungi that Infect Humans

5. Willey, Joanne M; Sherwood, Linda; Woolverton, Christopher J; Prescott, Lansing M. Microbiology : Prescott, Harley, and Klein's microbiology : Publication date 2008 : Publisher New York : McGraw-Hill Higher Education : Collection folkscanomy; additional_collections

6. Gerard Tortora, Berdell Funke, Christine Case, Derek Weber , Warner Bair III : Microbiology: An Introduction 13th Edition : Publisher : Pearson; 13th edition (January 8, 2018) : Hardcover : 960 pages

7. Sunil Dogra1, Dipika Shaw2, Shivaprakash M Rudramurthy2 : Antifungal drug susceptibility testing of dermatophytes: Laboratory findings to clinical implications 
8. Year : 2019 | Volume : $10 \mid$ Issue : 3 | Page : 225-233 Publication of IADVL in India

9. medical editors : Mayo Clinic Marketplace : Nail fungus : June 09, 2011.

10. The Inhibition Effect of some Plant's extracts on growth of dermatophytes isolation fro $\mathrm{m}$ patients : Qadory, A. G. : 2008 : College of Science. Uni. of Tikrit. Iraq.

11. Superficial fungal infections seen at the National Skin Tan H. H (2005). Centre, Singapore 